\title{
PDGFRA EXPRESSION IN ADULT ASTROCYTOMA AND ITS RELATION TO CELL CYCLE REGULATORS; P53 AND CYCLIN D1
}

\author{
$\mathscr{B} 8 y$ \\ Afaf. T. Ibrahiem*, Hoda Saleh ** \\ \& Shaimaa M Yussif*** \\ Framam \\ Department of Pathology* Faculty of Medicine, Mansoura University
}

\begin{abstract}
Background: Over the past decade there have been several attempt tries for molecular classification of CNS tumors, also to identify biological markers that reflect the degree of tumour malignancy. Such markers might assist in identifying tumors with a poor prognosis and application of new medical target therapy ${ }^{2}$. Platelet-derived growth factor (PDGF) signalling has been shown to be a key regulator of glioma development. Clinical trials evaluating the efficacy of anti-PDGFRA therapies on gliomas are ongoing 31 .

Objectives: evaluation of PDGFRA protein in adult astrocytoma as a new hope to use PDGFRA as biological prognostic marker and possi-
\end{abstract}

bility of using anti PDGFRA as a target therapy in astrocytoma. Also we aimed to correlate expression of PDGFRA protein to p53 and cyclin D1 expression in astrocytoma.

Material \& Methods: well representative paraffin embedded blocks of 57 cases of adult astrocytoma (16 diffuse astrocytoma, 9 anaplastic astrocytoma, 28 cases were GBM, and 4 cases were gliosarcoma) were evaluated for PDGFRA protein, P53, Cyclin D1 expression. Correlation of level of expression of these proteins were correlated to clinicopathologic criteria was done

Results: According to WHO, 16 Cases were grade II, 9 cases were grade III, while 32 had grade IV.

MANSOURA MEDICAL JOURNAL 


\section{PDGFRA EXPRESSION IN ADULT ASTROCYTOMA etc...}

PDGFRA was positive in all cases of astrocytoma cases. with varied interisty PDGFRA expression was also observed in tumour-associated endothelial cells of blood vessels in approximately $8 \%$ of the cases. PDGFRA showed significant correlation with histopathological type ( $p$ value < 0.001). Also PDGFRA was significantly correlated to WHO grading of the tumor ( $p$ value $<0.001$ ) with no significant correlation to age, gender or site of tumor. 43 cases (>109\%) expressed cyclin D1 but with varied intensity of expression. Cyclin D1 showed significant correlation with anatomical site of tumor $(p$ value $=0.032)$, with age ( $p$ value $=0.003$ ), however, no significant correlation to gender. $82.5 \%$ of cases expressed p53, P53 showed significant correlation with histopathological type $(p$ value $=0.001)$.

\section{INTRODUCTION}

Primary CNS tumors are one of the deadliest human cancers. Gliomas are the most common form of primary malignancies of the central nervous system (CNS) account for approximately $70 \%$ of all primary brain tumours 1,2

Vol. 44, No. 1 \& 2 Jan. \& April, 2015
Astrocytoma is the most common primary neoplasm of the central nervous system, representing $30 \%$ to $40 \%$ of all tumors. Based on histological, immunohistochemical, and ultrastructural criteria, the astrocytic tumors are graded are graded on a scale from I-IV 3,2

According to WHO. (2007), diffusely infiltrative astrocytic tumours are classified as diffuse astrocytomas (grade II) which are slowly growing, diffusely infiltrating astrocytomas that are prone to progress to higher grades. Anaplastic astrocytomas (grade III) display increased proliferation rate, increased cellularity and nuclear atypia. Glioblastoma multiforme (grade IV) is the most malignant form of astrocytoma characterized by microvascular proliferation, necrosis and cellular pleomorphism²

The high-grade tumors can present de novo as primary glioblastomas, or evolve through progression from lower-grade tumors as secondary glioblastomas by gaining more mutations. The primary and secondary glioblastomas show the same histopathological features, al- 
though they differ in both genetic ab- identify biological markers that reerrations and the clinical history $4 . \quad$ flect the degree of tumour malignanMolecular genetic analyses have revealed that the progression of astrocytic tumors results from accumulating inactivation of different tumor suppressor genes and/or amplification of certain oncogenes 5 cy. Such markers might assist in identifying tumors with a poor prognosis and application of new target therapy 10

\section{PDGFRA:}

The family of platelet-derived

The prognosis of glioma is poor, Histologic grading has been regarded as one of the most important predictors of outcome in patients with astrocytomas but this assessment is subjective and depends on the experience and training of individual pathologists. Also progression of low grade glioma rapidly to higher while other cases progress slowly remain unexplained 6,7

Treatment options for glioma including strocytoma are surgical resection, radiation therapy and chemotherapy8. However, with these aggressive treatments, patient response is poor and the aggressive glioma patient's survival is less than 2 years 9

Over the past decade there have been several attempts for molecular classification of CNS tumors, also to growth factors (PDGFs) and their receptors are involved in normal embryonic development, cellular differentiation, and response to tissue damage. Aberrant activity of the PDGFs and their receptors are involved in pathological conditions as inflammation, atherosclerosis, and cancer. The PDGF is recognized by 2 types of cell surface receptors, that is, PDGFRA and PDGFRB 11, 12

PDGFRA and PDGFRB belong to class III family of receptor tyrosine kinases (RTKs). PDGFRA and its main ligand PDGFA are key regulators of glial cells proliferation, mainly oligodendrocytes, and have an important role in normal development of the central nervous system 13

Mutation in PDGF receptor genes have been found in gastrointestinal stromal tumors (GIST) and certain

MANSOURA MEDICAL JOURNAL 
leukemias 11,15,16. Lokker et al. $2002^{17}$ reported that overexpression of PDGF ligands and their receptors particularly PDGFRA are expressed in glial tumors through activating RAS and PI3 kinase signaling pathways. PDGFRA gene amplification has been reported in glioblastomas $^{18}$

Growth stimulation by PDGF is associated with the pathway of secondary glioblastomas, PDGF receptor profile may be a biomarker for a subgroup of GBM originating from $A$ PDGF receptor-responsive cell however formal proof is still missing. Also, the PDGFR? gene is amplified in a subset of GBM, this strongly indicates that it confers a selective growth advantage and has a mechanistic role in the pathogenesis of the tumor 19

Treatment modalities involving genomic profiling and personalized medicine are expected to improve the therapy targeting PDGFR? signaling. the availability of clinically useful small-molecule inhibitors, such as imatinib mesylate (Glivec) and sunitinib (Sutent) has increased the interest of using PDGFR as a Vol. 44, No. 1 \& 2 Jan. \& April, 2015 cancer drug target 20,21

\section{Cyclin D1:}

Cyclins are proteins that are expressed during and regulate the normal progression of cell through the cell cycle. Over expression of different types of cyclins occurs in many cancers and in some of them, cyclins expression correlated with the grades of malignancy 22 . The deregulation of cyclin expression involved in carcinogenesis by causing proliferation and causing over-riding checkpoints that affect genomic integrity 23

Cyclin D1 is a proto-oncogene is located in the $11 q 13$ region and plays a positive-regulation role in the progression of cell cycle. from G1 to $S$ phase, this achieved by forming active complexes with cyclin dependent kinase 4 and 6 (CDK4 and CDK6) also cyclin D1 regulates the activity of several transcription factors 24. Amplification and overexpression of cyclin D1 occurs in several malignancies as breast cancer, lung cancer, melanoma and oral squamous cell carcinomas 25. In many cancers, Cyclin D1 overexpression is associated with shorter 
patient survival and increased metastasis. Cyclin D1, is considered as a biomarker of cancer phenotype and disease progression 26

The expression of cyclin $A$ and cyclinB1 proteins were detected in astrocytoma and showed relation to the histologic grade or proliferative activities. In astrocytomas Cyclin D1 expression is associated with cell proliferation activity and patient prognosis 27 . The cyclin D1-is normaly expressed in normal oligodendrocytes and microglia cells 28

Also due to the multiplicity of cyclin D effects on cancer cell biology, it considered a promising therapeutic target 24

\section{P53 protein}

P53 gene is a tumor suppressor gene. It located on the short arm of Chromosome 17 at band 13.1 and consists of 11 exons. It acts as a "molecular policeman" as preventing the proliferation of geneticaally damaged cells. It produces its effects by acting as a transcription factor, regulating thousands of genes that are involved in proliferation, apoptosis, DNA repair and angiogenesis. Also
P53 induce apoptosis of neurons and neural progenitors during development. P53 protein can be indirectly used for detection of p53 gene alterations by immunohistochemistry 29

P53 gene alterations had been detected in pediatric and adult astrocytic tumorigenesis. Also, P53 gene alterations have a critical role in the initiation, recurrence and progression of astrocytic tumors of Grades II, III and IV. in $87 \%$ of glioblastomas, genetic alterations in any of the components of the P53 signaling pathway has been found 30 Also, P53 gene was found to be inactivated in low-grade gliomas, indicating that loss of P53 is an early event in brain tumorigenesis 31

\section{MATERIALS AND METHODS}

\subsection{Case selection}

This retrospective study was carried out on 57 adult patients (35 male and 22 females) who underwent excisional biopsy for a primary CNS astrocytoma (GII, III, IV) received in our pathology department lab in the period from Jan 2012 to Jan 2014. None of the patients received any neoadjuvant chemothera- 
18

py or radiotherapy. Complete clinicopathological information were obtained from patient medical records in the archives of the pathology lab of Mansoura faculty of medicine

\subsection{Histopathological examina-} tion:

Tissue samples

Formalin-fixed paraffin-embedded blocks from fifty seven cases of glioma were retrieved from pathology tab archives of the Department of Pathology of Mansoura Univeristy. Sections of 4 um thickness have been cut from formalin fixed paraffin embedded blocks for routine H\&E, others were prepared on charged slides for immunohistochemistry. Slides were reviewed to dconfirm the histological type and WHO grading system according to Louis etal ${ }^{2}$.

Examination of three tumor slides from each specimen was done on an Olympus CX31 light microscope. Pictures were obtained by a PC-driven digital camera (Olympus E-620). Ethical approval of this study was not required by our institution as this study was based on retrospective analysis dealing with archival paraffin slides and blocks, not related to patient's privacy, impairment or treatment.

2.3. Immunohistochemical staining: Immunohistochemical staining for PDGFRA, P53 protein, and cyclin D1 with a labelled streptavidin- biotinperoxidase complex technique. Commercially available antibody against P53 (mouse monoclonal P53 antibody, isotype IgG2a, Genemed, dil 1:50), PDGFA ((Rabbit polycolonal, clone CB-11, Thermo scientific, 1:50 dilution, USA), cyclin D1 (Mouse monoclonal Anti-Human Cyclin D1 (Dako, dil 1:100 dilution). Detection kit used was high sensitive kit (DakoCytomation envision +dual link system peroxidase code K4061) using $D A B$ as chromogene. Antigen retrieval required pretreatment with 1 mM EDTA (at pH 8.0) for 20 minutes (P53, cyclin D1, and PDGFA) in microwave oven. Proper positive and negative controls were performed. Breast carcinoma for P53, papillary serous adenocarcinoma of ovary for PDGFRA. As a negative control, sections were stained without the addition of a primary antibody.

2.4. Immunohistochemical interpretation

As for the immunohistochemistry 
assessment, Slides were scanned by $\quad=$ negative cells ( $0 \%$ of positive cells) X40 magnification. Ten cellular are33 as were selected (i.e. the so-called hot spots) and evaluated at $\mathrm{X} 400$ magnification.

For PDGFA expression: semiquntative 4-score intenisty scale was used as follow (-) (negative), (+) (?5\%), (++) (5-50\%), and (+++) $(>50 \%)$. Samples with scores $(-)$ and $(+)$ were considered negative, and those with scores $(++)$ and $(+++)$ were considered positive PDGFRA staining was confined to cytoplasm and membrane32 (Martinho et al., 2009).

For P53 expression: Positive p53 protein staining was defined as nuclear staining, cytoplasmic staining was considered nonspecific and ignored. The percentage of tumor cell nuclei with positive staining was evaluated in relation to total number of neoplastic nuclei in at least 10 fields observed at magnification $x$ 400. Scoring was categorized follows: $3+=$ high level $(91-100 \%$ of positive cells), $2+=$ medium level (11-90\% of positive cells), $1+=$ low level (up to $10 \%$ of positive cells), -

Cyclin D1 protein expression: immunostained tumor cells were counted in 5 consecutive microscopic fields (magnification 100x) per tumor sample in area of highest density of staining. In each field, 100 tumor cell nuclei were evaluated and the mean for every 5 fields were calculated. the positive staining is granular and nuclear. Scoring was categorized;1 $<5 \%$ immunopositive cells was considered negative, and $2>5 \%$ immunopositive cells was considered positive. Samples from patients with $<50 \%$ cyclin D1 were considered low expressors, whereas those with 3 $>50 \%$ cyclin D1 positive tumor cells were considered high expressors 34

\subsection{Statistical analysis:}

The statistical analysis of data was done by using excel(Microsoft Office 2013) program and SPSS (statistical package for social science) program (SPSS, Inc, Chicago, IL) version 20. Kolmogorov-Smirnov test was done to test the normality of data distribution. Qualitative data were presented as frequency and percentage. Chi square test was

MANSOURA MEDICAL JOURNAL 
20

used to compare groups. Quantitative data were presented as mean, standard deviation. For comparison of more than two groups; One-Way ANOVA (for parametric data) was used. $p$ is significant if $\leq 0.05$ at confidence interval $95 \%$.

\section{RESULTS}

3.1. Clinicopathologic features: (table 1)

Fifty seven patients with adult astrocytoma were studied retrospectively. They were admitted to the Mansoura University hospital for primary surgical treatment between January 2012 and January 2014. The age of the patients ranged from 24 to 72 years with a mean age of 50.84 years \pm 11.25 . Out of 57 patients, $35(61.4 \%)$ were males and $22(38.6 \%)$ were females. Regarding the site of tumor 14 were frontal, 10 were parietal, 12 were temporal, 18 were temproparietal, and 3 were thalamus ( table 1)

Histologically, 16 cases (66.7\%) were diffuse astrocytoma two of them showed gemistocytic differentiation. $9(29.2 \%)$ were anaplastic astrocytoma including 2 with gemistocytic astrocytoma. 28 cases were GBM (16.7\%), and 4 cases were gliVol. 44, No. 1 \& 2 Jan. \& April, 2015 osarcoma. According to WHO, 16 Cases were grade II, 9 cases were grade III, while 32 had grade IV

3.2. Immunohistochemical analysis:

PDGFRA expression: (table 2, table 3)

All cases expressed PDGFRA but with varied intensity of expression. PDGFRA showed significant correlation with anatomical site of tumor ( $p$ value $=0.032$ )

PDGFA expression was also observed in tumour-associated endothelial cells and in basment membrane of blood vessels in approximately $8 \%$ of the cases (Figure 1). PDGFRA showed significant correlation with histopathological type ( $p$ value $<0.001$ ) (Table 3 ). $37.6 \%$ of diffuse fibrillary astrocytoma expressed PDGFA mainly of score $1(36.3 \%)$, one case of diffuse fibrillary astrocytoma expressed PDGFA of score 2 and histologically this case showed gemistocytic differentiation. All cases of anaplastic astrocytoma expressed PDGFRA ranged from 1 to 3 with $36.4 \%$ of cases considered positive (score 2). Regarding GBM all cases expressed PDGFRA (from 1 to 3 ) as $82.1 \% \%$ of 
GBM were of score 3 and 4). All cas- $\quad=0.001)$. P53 expression showed no es of gliosarcoma were positive, of score 3 and 4. Also PDGFA showed statistically significant correlation to WHO grading of the tumor ( $p$ value $<0.001$ ). PDGFRA expression showed no significant correlation to age, gender or site of tumor. PDGFRA expression showed significant correlation to P53 expression ( $p$ value $<0.001$ )

P53 expression: (table 2, table 4)

The immunostaining for P53 was detected in the nuclei. $82.5 \%$ of cases expressed p53 (figure 2), P53 showed significant correlation with histopathological type ( $p$ value $=$ 0.001) Diffuse astrocytoma expressed p53 of score ranging from 0 to 2 , score 2 mainly expressed in diffuse astrocytoma with gemistocytic differentiation. Anaplastic astrocytoma expression of p53 ranged from 1 to 3. Regarding GBM it showed wide range of $p 53$ expression (from 0 to 3) as $21.1 \%$ of GBM cases didn't express p53 while $42.9 \%$ highly expressed p53. Seventy five percent of gliosarcoma cases highly expressed p53 (score 3). Also p53 expression was significantly correlated to WHO grading of the tumor ( $p$ value significant correlation to age, gender or site of tumor

Cyclin D1 expression (table 2, table 5):

The immunostaining for cyclin D1 was detected in the nuclei. $71.9 \%$ of cases expressed cyclin D1 but with varied intensity of expression (figure 3). Cyclin D1 showed significant correlation with anatomical site of tumor ( $p$ value $=0.032$ ), with age ( $p$ value $=0.003$ ), however, no significant correlation to gender. Cyclin D1 expression showed significant correlation with histopathological type ( $\mathrm{p}$ value $<$ 0.001 ). Only $14.3 \%$ of diffuse astrocytoma cases expressed Cyclin D1 and were of low expressers score. All cases of anaplastic astrocytoma expression of cyclin D1 with of $71.4 \%$ of cases are high expressers. Regarding GBM, $89.2 \%$ of cases expressed Cyclin D1 with $57.1 \%$ of GBM were high expressers. Like GBM, all gliosarcoma cases were positive for cylin D1 with $75 \%$ were of high expression for cyclin D1. Also, Cyclin D1 was significantly correlated to WHO grading of the tumor ( $p$ value $<0.001$ )

MANSOURA MEDICAL JOURNAL 
Table 1: Clinical and histopathological criteria of the studied astrocytoma cases:

\begin{tabular}{|c|c|c|c|}
\hline \multicolumn{2}{|l|}{ age } & 50.84 & 11.25 \\
\hline \multirow{2}{*}{ Sex } & Male & 35 & $61.4 \%$ \\
\hline & Female & 22 & $38.6 \%$ \\
\hline \multirow{5}{*}{ Clinically } & Frontal & 14 & $24.6 \%$ \\
\hline & Parital & 10 & $17.5 \%$ \\
\hline & Temporal & 12 & $21.0 \%$ \\
\hline & Temproparietal & 18 & $31.6 \%$ \\
\hline & Thalamus & 3 & $5.3 \%$ \\
\hline \multirow{5}{*}{ Histopathology } & Diffuse astrocytoma & 14 & $24.6 \%$ \\
\hline & GBM & 28 & $49.1 \%$ \\
\hline & Gemistocytic diffuse astrocytoma & 2 & $3.5 \%$ \\
\hline & Anaplastic astrocytoma & 9 & $15.8 \%$ \\
\hline & Gliosarcoma & 4 & $7.0 \%$ \\
\hline \multirow{3}{*}{ Grade } & II & 16 & $28.1 \%$ \\
\hline & III & 9 & $15.8 \%$ \\
\hline & IV & 32 & $56.1 \%$ \\
\hline
\end{tabular}

Table 2: Biological markers (p53, PDGFRA, cyclin D1) expression in adult astrocytoma cases:

\begin{tabular}{|l|l|r|r|}
\hline Oncogene & Score & No of cases & $\%$ \\
\hline \multirow{5}{*}{ P53 } & 0 & 10 & $17.5 \%$ \\
\cline { 2 - 4 } & 1 & 12 & $21.1 \%$ \\
\cline { 2 - 4 } & 2 & 17 & $29.8 \%$ \\
\cline { 2 - 4 } & 3 & 18 & $31.6 \%$ \\
\hline \multirow{5}{*}{ PDGFA } & 1 & 9 & $18.5 \%$ \\
\cline { 2 - 4 } & 2 & 15 & $26.3 \%$ \\
\cline { 2 - 4 } & 3 & 24 & $42.1 \%$ \\
\cline { 2 - 4 } & 4 & 9 & $18.5 \%$ \\
\hline \multirow{3}{*}{ CYCLIN D1 } & negative & 16 & $28.1 \%$ \\
\cline { 2 - 4 } & Low expression & 17 & $29.8 \%$ \\
\cline { 2 - 4 } & High expression & 24 & $42.1 \%$ \\
\hline
\end{tabular}

Vol. 44, No. 1 \& 2 Jan. \& April, 2015 
Afaf.T.Ibrahiem et al ...

Table 3: Correlation between PDGFRA and histopathological criteria of studied 57 astrocytoma cases:

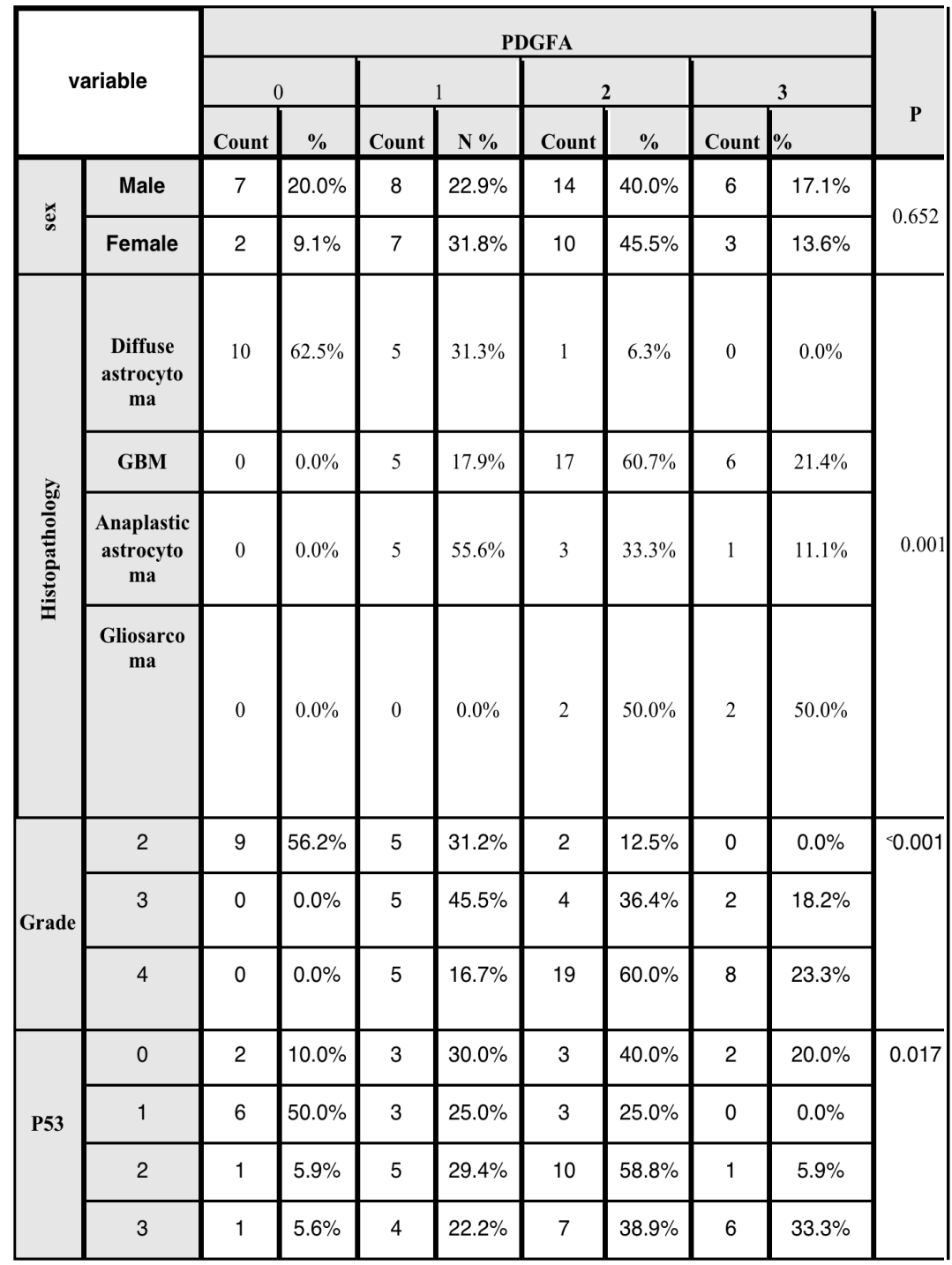

MANSOURA MEDICAL JOURNAL 
Table 4: Correlation between P53 and histopathological criteria of studied 57 astrocytoma cases:

\begin{tabular}{|c|c|c|c|c|c|c|c|c|c|c|}
\hline & & \multicolumn{8}{|c|}{ P53 } & \multirow{3}{*}{$\mathbf{P}$} \\
\hline & & \multicolumn{2}{|c|}{0} & \multicolumn{2}{|c|}{1} & \multicolumn{2}{|c|}{2} & \multicolumn{2}{|c|}{3} & \\
\hline & & Count & $\%$ & Count & N \% & Count & $\%$ & Count & $\%$ & \\
\hline \multirow{2}{*}{ 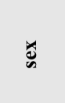 } & Male & 6 & $17.1 \%$ & 8 & $22.9 \%$ & 7 & $20.0 \%$ & 14 & $40.0 \%$ & \multirow{2}{*}{0.692} \\
\hline & Female & 4 & $18.2 \%$ & 4 & $18.2 \%$ & 6 & $27.3 \%$ & 8 & $36.4 \%$ & \\
\hline \multirow{5}{*}{$\frac{\Xi}{n}$} & $\mathbf{F}$ & 2 & $14.3 \%$ & 2 & $14.3 \%$ & 3 & $21.4 \%$ & 7 & $50.0 \%$ & \multirow{5}{*}{0.481} \\
\hline & $\mathbf{P}$ & 2 & $20 \%$ & 2 & $20 \%$ & 4 & $40 \%$ & 2 & $20 \%$ & \\
\hline & $\mathbf{T}$ & 3 & $25 \%$ & 2 & $16.7 \%$ & 4 & $33.3 \%$ & 3 & $25 \%$ & \\
\hline & $\mathbf{T} / \mathbf{P}$ & 5 & $27.8 \%$ & 4 & $22.2 \%$ & 6 & $33.3 \%$ & 3 & $16.7 \%$ & \\
\hline & Thalamus & 0 & $0 \%$ & 2 & $66.7 \%$ & 0 & $0.0 \%$ & 1 & $33.3 \%$ & \\
\hline \multirow{4}{*}{ 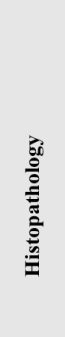 } & $\begin{array}{c}\text { Diffuse } \\
\text { astrocytoma }\end{array}$ & 3 & $18.8 \%$ & 7 & $43.8 .0 \%$ & 6 & $37.5 .4 \%$ & 0 & $0 \%$ & \multirow{4}{*}{0.001} \\
\hline & GBM & 7 & $25 \%$ & 2 & $7.1 \%$ & 9 & $32.3 \%$ & 10 & $35.7 \%$ & \\
\hline & $\begin{array}{c}\text { Anaplastic } \\
\text { astrocytoma }\end{array}$ & 0 & $0.0 \%$ & 2 & $22.2 \%$ & 1 & $11.1 \%$ & 6 & $21.4 \%$ & \\
\hline & Gliosarcoma & 0 & $0.0 \%$ & 1 & $25 \%$ & 1 & $25 \%$ & 2 & $50 \%$ & \\
\hline \multirow{3}{*}{ Grade } & 2 & 2 & $12.5 \%$ & 9 & $56.3 \%$ & 5 & $31.3 \%$ & 0 & $0.0 \%$ & \multirow[t]{3}{*}{$<0.001$} \\
\hline & 3 & 0 & $0 \%$ & 2 & $22.2 \%$ & 5 & $55.5 \%$ & 2 & $22.2 \%$ & \\
\hline & 4 & 8 & $25 \%$ & 1 & $3.1 \%$ & 7 & $21.9 \%$ & 16 & $50 \%$ & \\
\hline
\end{tabular}

Vol. 44, No. 1 \& 2 Jan. \& April, 2015 
Table 5: Correlation between cyclin D1 and histopathological criteria of studied 57 astrocytoma cases:

\begin{tabular}{|c|c|c|c|c|c|c|c|c|}
\hline & \multirow{3}{*}{ Variable } & \multicolumn{7}{|c|}{ Cyclin D1 } \\
\hline & & \multicolumn{2}{|c|}{1} & \multicolumn{2}{|c|}{2} & \multicolumn{2}{|c|}{3} & \\
\hline & & Count & $\%$ & Count & N \% & Count & $\%$ & \\
\hline \multirow{2}{*}{ 苛 } & MALE & 11 & $31.4 \%$ & 12 & $34.3 \%$ & 12 & $34.3 \%$ & \multirow{2}{*}{0.319} \\
\hline & FEMALE & 5 & $22.7 \%$ & 5 & $22.7 \%$ & 12 & $54.5 \%$ & \\
\hline \multirow{5}{*}{ 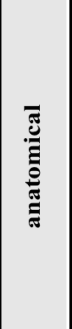 } & Frontal & 5 & $35.7 \%$ & 4 & $28.6 \%$ & 5 & $42.9 \%$ & \multirow{5}{*}{0.851} \\
\hline & Parietal & 2 & $20 \%$ & 2 & $20 \%$ & 6 & $60 \%$ & \\
\hline & Temporal & 4 & $27.3 \%$ & 3 & $27.3 \%$ & 5 & $45.5 \%$ & \\
\hline & Temproparietal & 5 & $27.8 \%$ & 7 & $38.9 \%$ & 6 & $33.3 \%$ & \\
\hline & Thalamus & 1 & $33.3 \%$ & 1 & $33.3 \%$ & 1 & $33.3 \%$ & \\
\hline \multirow{4}{*}{ 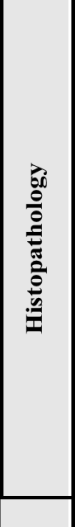 } & $\begin{array}{c}\text { Diffuse } \\
\text { astrocytoma }\end{array}$ & 12 & $85.7 \%$ & 2 & $14.3 \%$ & 0 & $0.0 \%$ & \multirow{4}{*}{0.001} \\
\hline & GBM & 3 & $10.7 \%$ & 9 & $32.1 \%$ & 16 & $57.1 \%$ & \\
\hline & $\begin{array}{c}\text { Anaplastic } \\
\text { astrocytoma }\end{array}$ & 0 & $0.0 \%$ & 4 & $28.6 \%$ & 5 & $71.4 \%$ & \\
\hline & Gliosarcoma & 0 & $0.0 \%$ & 1 & $25.0 \%$ & 3 & $75.0 \%$ & \\
\hline \multirow{3}{*}{ Grade } & 2 & 13 & $81.2 \%$ & 3 & $18.8 \%$ & 0 & $0.0 \%$ & \multirow[t]{3}{*}{$<0.001$} \\
\hline & 3 & 0 & $0.0 \%$ & 4 & $45.5 \%$ & 5 & $54.5 \%$ & \\
\hline & 4 & 3 & $10.0 \%$ & 10 & $30.0 \%$ & 19 & 60.0 & \\
\hline
\end{tabular}

MANSOURA MEDICAL JOURNAL 


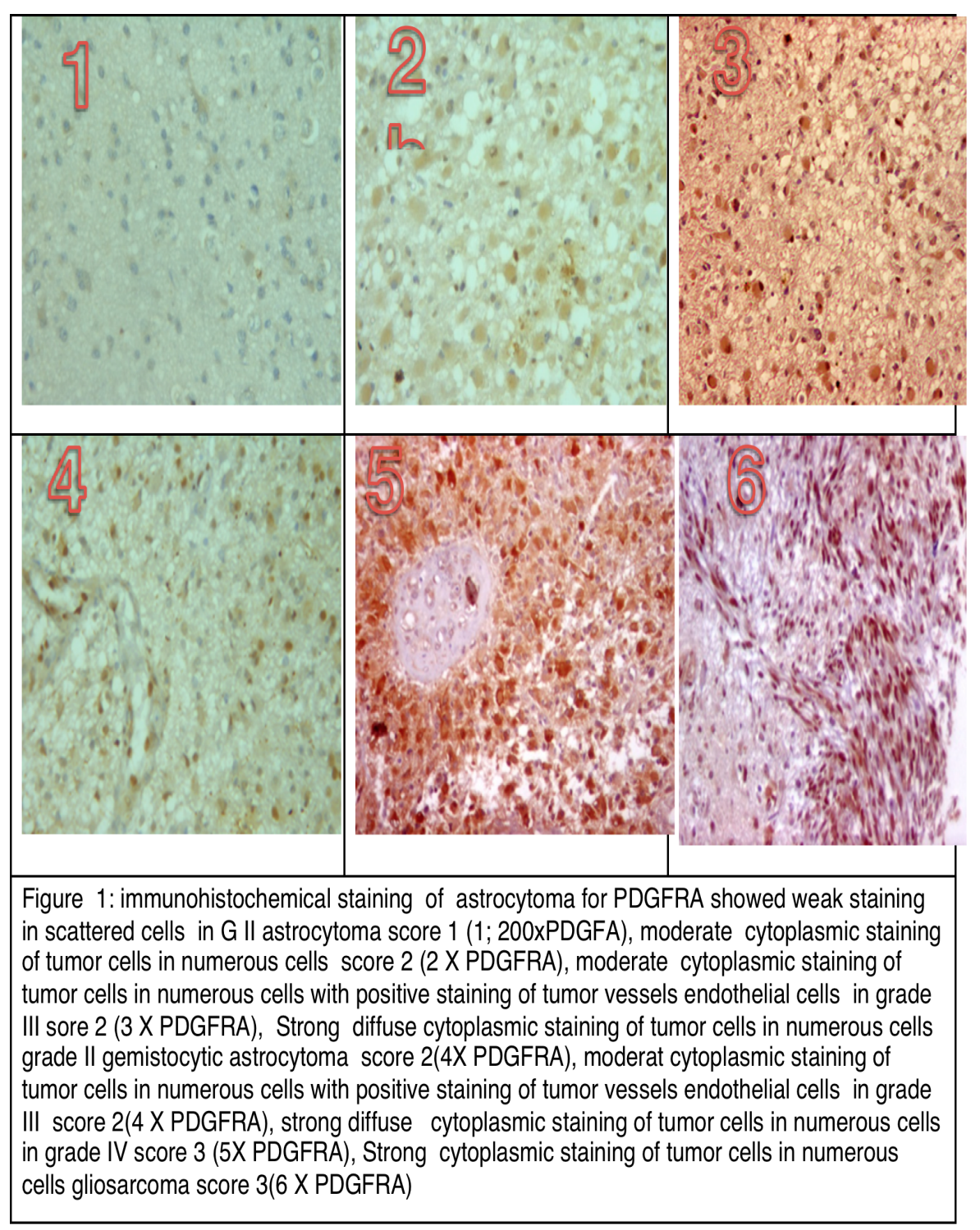

Vol. 44, No. 1 \& 2 Jan. \& April, 2015 


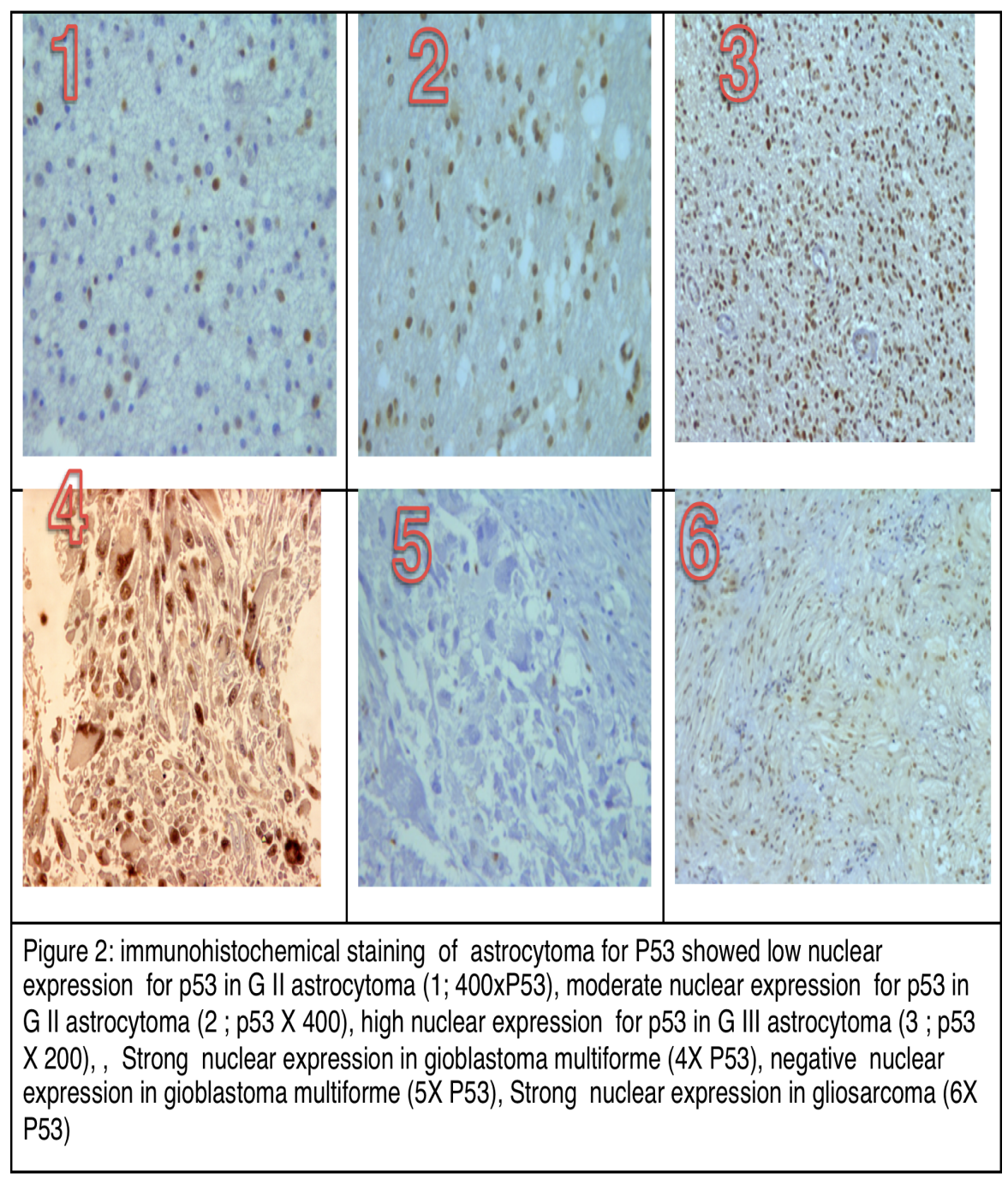

MANSOURA MEDICAL JOURNAL 


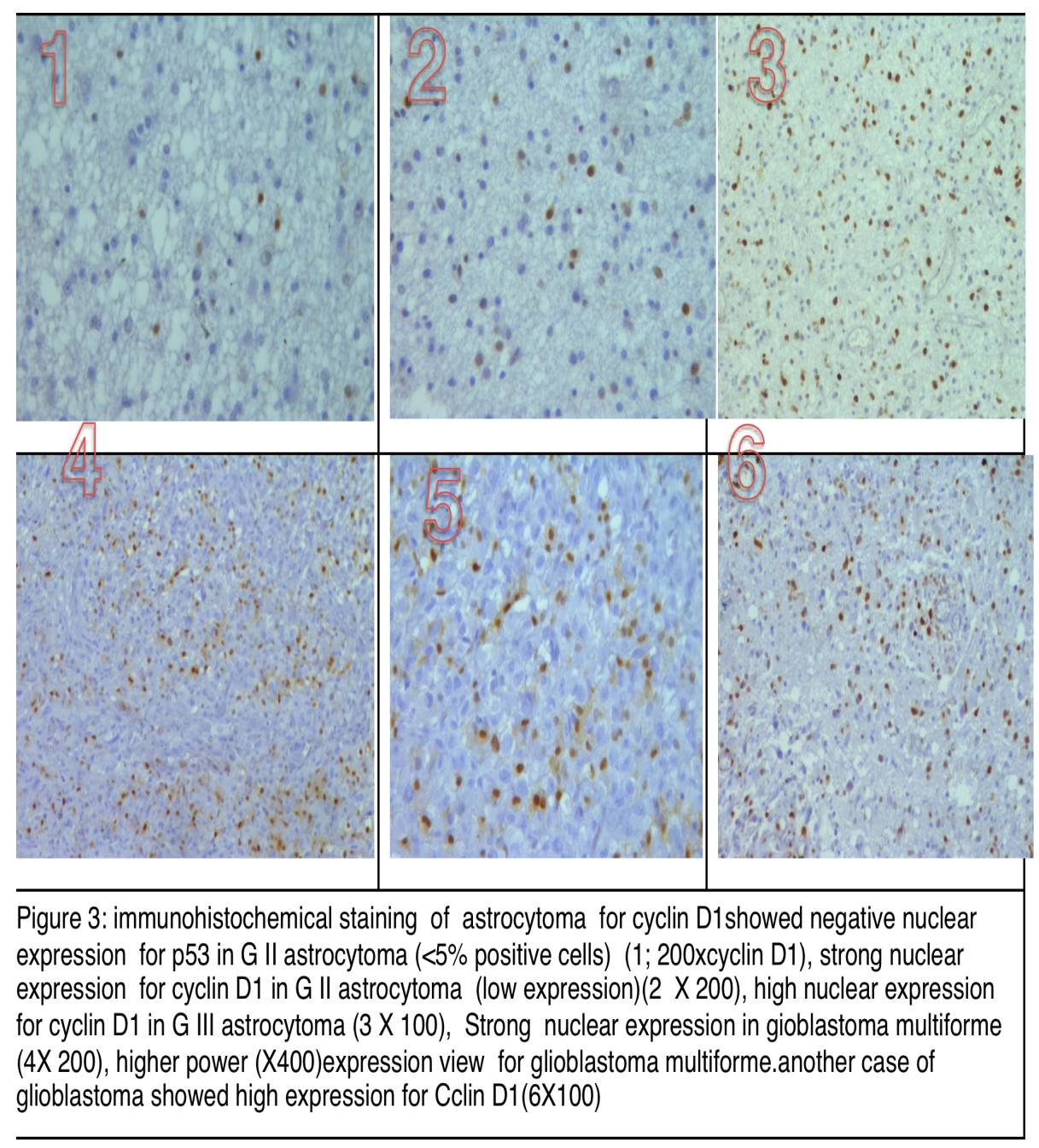

Vol. 44, No. 1 \& 2 Jan. \& April, 2015 


\section{DISCUSSION}

The histological subtyping and the grading of gliomas in the World Health Organization Classification (WHO) of brain tumours still the most the most reliable, accepted indicator for the clinical outcome in adult and pediatric glioma patients. However, it has also become clear that tumours virtually identical by morphology may have very different outcomes. So, molecular and biological markers may aid in deriving more detailed prognostic information $35, f^{5}$ Traditionally, the higher the grade of gliomas, the worse the prognosis 2

Molecularly targeted therapies have entered clinical trials for brain tumors. The availability of clinically useful small-molecule inhibitors, such as imatinib mesylate (Glivec) and sunitinib (Sutent) has increased the interest of using PDGFR as a cancer drug target 21,36 .

In the present study, we screened for PDGFRA expression in 16 patients with low-grade diffuse astrocytomas, 9 patients with anaplastic astrocytoma and 28 glioblastoma multiforme and 4 with gliosarcoma and correlated these data with other biological markers that regulate the cell cycle progression. Also correlated PDGFRA expression with clinicopathologic features

The PDGF pathway is involved in glial tumorigenesis including cellular proliferation, migration, development, and angiogenesis 37,38,39. PDGFRA was positive in all of our series of astrocytoma cases. Martinho, et al., 200932 found that PDGFRA expression was detected in $29.6 \%$ of gliomas, also they reported that $60 \%$ of malignant astrocytic tumours expressed PDGFRA. Also, previous studies reported approximately $50 \%$ of malignant astrocytomas expressed PDGFRA40, The correlation between Ribom et al, 200241 reported a favorable prognosis of glioma associated with PDGFRA expression

There was evidence for involvement of the overexpression of the PDGFA receptor (PDGFR) is considered to be an early event in the progression of low grade gliomas. This finding is consistent with our observation of low expression of PDGFA in low grade astrocytoma and increasing expression with high grade astrocytoma 42 .

MANSOURA MEDICAL JOURNAL 
High expression of PDGFRA in glioblastoma multiforme $(82 \%$ express score 3 and 4). This confirmed by that is concluded by Westermark. 201419 of a mechanistic role PDGFRA in the pathogenesis of the and progression of tumor

Also in some high grade Astrocytoma cases $(8 \%)$ of the present study series showed positive staining of the tumor blood vessels endothelial cells. This provide evidence for additional role of PDGFRA in tumor angiogenesis 38

In our study more expression of PDGFRA occurred in grade I and II astrocytoma with gemistocytic differentiation. This finding is in opposite to that founded by Ribom et al. 41 who reported that PDGFR $\alpha$ expression did not differ among fibrillary, gemistocytic, and protoplasmic astrocytomas. Overexpression of PDGF receptors in tumors has has led to the development PDGF/PDGF receptor antagonists to treat patients with various malignancies 43

Also, Shen et al. ${ }^{43}$ reported that glioma stem cells preferentially express PDGFR? and its activation promotes glioma stem cell self- renewal. This may explain a high rate of recurrence and resistance to treatment is in malignant gliomas 44

In the present study, PDGFB showed significant correlation with anatomical site of tumor. PDGFB showed significant correlation with histopathological type ( $p$ value < 0.001 ). Most of the previous studies were experimental and concerned with role of PDGFR in pathogenesis of glioma especially glioblastoma $45,46,39$. PDGFRA expression in 24 $(69 \%)$ of 35 of diffuse astrocytomas, although high-level amplification was not detectable by quantitative polymerase chain reaction (PCR) 47

Mutation of the p53 gene and p53 protein overexpression were found in $30-60 \%$ of astrocytic gliomas regardless of the tumour grade 48,49 . TP53 mutations have been considered the most frequent and earliest genetic alteration in patients with WHO grade II astrocytomas 50

In this study, 47 cases $(82.5 \%$ of cases expressed p53). These results cope with Ranuncolo et al ${ }^{51}$ who reported $66 \%$ of their cases of glioma expressed $p^{53}$. This difference may be explained by increased number of 
secondary glioblastoma that occurred on top of diffuse or anaplastic astrocytoma and this may be due difference in modality treatment

Also, we found expression of p53 was correlated to histopathological typing of adult glioma and $\mathrm{WHO}$ grading as there was increasing p53 expressin with higher grades of the tumor. This was in concordance with Ranuncolo et al 51 .

Martinho et al 31 concluded that TP53 are frequently mutated and represent early events in the pathogenesis of WHO grade II astrocytomas/oligoastrocytomas. However in other study carried by Jiang et al, there was no correlation between the frequencies of p53 immunopositivity with the tumor grade 52

Cyclin D1 has been considered to be a cell cycle regulator of G1-S progression in the cell cycle its overexpression has been observed in many human tumors as breast, rectal 53 oesophageal 54 and head and neck carcinoma 55

The multiplicity of cyclin D effects on cancer cell biology, and evidence for their CDK-independent actions on end points such as cell migration and the DNA damage response, So, it can be considered an attractive therapeutic target 56,57 .

In the current study, we evaluated expression of Cyclin D1 in Adult astrocytoma; we found that there was significant correlation between percentage of nuclear expression of cyclin D1 and histopathological types and grading of the astrocytoma. High grade glioma showed high expression and there was overlap in expression scores between anaplastic astrocytoma and glioblastoma

The results of the study performed by 58 Cavalla et al cope with the results of the present study as he recorded that cyclin D1 protein expression had been shown to increase with histologic grade malignancy in astrocytomas. Also, Tan et al 59 results agreed with ours as they reported that there was significant difference in cyclin D1 expression between low and high grade glioma groups and concluded that the expression of cyclin D1 can act as a biological marker in evaluating malig-

MANSOURA MEDICAL JOURNAL 
nancy of gliomas and prognosis of patients

The increased number of cycD1 positive nuclei in gliomas does not necessarily imply amplification of theCCND1 locus as the transition from $\mathrm{G} 1$ to $S$ phase is regulated not only by cycD1, but also by p16, cdk4, pRb and other proteins 60. Also other molecular alteration rather than cyclin D1 gene amplification are idenfied.

The expression of cyclin D1 showed heterogeneity throughout the same tumour with highest expression in invasive areas. This heterogeneity could be explained by variability in proliferative activity of the cells 58

In this study, there was significant correlation between cyclin D1 expression and p53 expression throughout the grades of glioma. These results cope with evidence of indicating that the combination of PDGFR signaling and loss of p53 function may be an early event in gliomagenesis 61. Also cope with Mishina $T$ et al 62 who reported that cyclin D1 expression significantly correlated with altered p53 protein expression

\section{Summary \& Recommendation}

Primary CNS tumors are one of the deadliest human cancers. Gliomas are the most common form of primary malignancies of the central nervous system (CNS) account for approximately $70 \%$ of all primary brain tumours. astrocytoma is the most common primary neoplasm of the central nervous system, representing between $30 \%$ to $40 \%$ of all tumors.

Treatment options for strocytoma are surgical resection, radiation therapy and chemotherapy. ${ }^{8}$. However, with these aggressive treatments, patient response is poor and the aggressive glioma patient's survival is less than 2 years. Over the past decade there have been several attempts for molecular classification of CNS tumors, also to identify biological markers that reflect the degree of tumour malignancy.

In this study, we evaluated 57 cases of adult astrocytoma for PDGFRA protein expression Also we correlated the expression of PDGFRA protein to p53 and cyclin 
D1 proteins expression in astrocytoma cases.

This study revealed positive staining for PDGFRA in (100\% all cases), 82.5\% for p53. $71.9 \%$ for cyclin D1. PDGFRA expression showed significant correlation with histopathological type. Also PDGFRA expression was significantly correlated to $\mathrm{WHO}$ grading of the tumor with no significant correlation to age, gender or site of tumor. Also, there was significant correlation between P53, cyclin D1 proteins expression and grading of astrocytoma.

PDGFRA protein is widely expressed in all grades of adult astrocytoma with high expression in glioblastoma, this provides more evidence for involvement of PDGF pathway in pathogenesis of astrocytoma and progression to higher grades. Our results may provide a step forward in the identification of a subset of astrocytoma which is PDGFRA positive that can be benefit from widely available anti PDGFRA target therapy. Further studies for correlation of PDGFRA protein expression with patient outcomes are recommended

\section{REFERENCES}

1. Furnari FB, Fenton T, Bachoo RM, Mukasa A, et al. (2007) : Malignant astrocytic glioma: genetics, biology, and paths to treatment. Genes Dev.; 21:2683-710.

2. Louis D,1 Ohgaki H, Wiestler O, Cavenee $\mathbf{W}$, et al. (2007) : The 2007 WHO Classification of Tumours of the Central Nervous System. Acta Neuropathol; Aug; 114(2): 97-109.

3. Kleihues P, D. N. Louis, B. W. Scheithauer et al., (2002) : "The WHO classification of tumors of the nervous system," Journal of Neuropathology and Experimental Neurology, vol. 61, no. 3, pp. 215-225,.

4. Ohgaki $\mathbf{H}$ and Kleihues $\mathbf{P}$. (2007) : Genetic Pathways to Primary and Secondary Glioblastoma. Am J Pathol.; 170(5): 1445-1453.

5. Collins VP (1995) : Genetic alterations in gliomas. J Neurooncol.; 24: 37-38

MANSOURA MEDICAL JOURNAL 
34 PDGFRA EXPRESSION IN ADULT ASTROCYTOMA etc...

6. Kim TS, Halliday AL, HedleyWhite ET, Convery K. (1991) : Correlates of survival and Daumas-Duport grading system for astrocytomas. J Neurosurg.; 74:27-37.

7. Suna $Y$, Zhangb $W$, Chenc $D$, Lva Y, et al., (2014) : A glioma classification scheme based on coexpression modules of EGFR and PDGFRA. PNAS.; 111 (9): 3539.

8. McCarthy BJ, and Kruchko C. (2005) : Consensus conference on cancer registration of brain and central nervous system tumors. Neuro Oncol.; 7:196-201.

9. Phillips HS, Kharbanda $S$, Chen R, Forrest WF, et al. (2006) : Molecular subclasses of high-grade glioma predict prognosis, delineate a pattern of disease progression, and resemble stages in neurogenesis. Cancer Cell.; 9:157-173.

10. Vigneswaran $K$, Neill $S$, and Vol. 44, No. 1 \& 2 Jan. \& April, 2015
Hadjipanay C. (2015) : Beyond the World Health Organization grading of infiltrating gliomas: advances in the molecular genetics of glioma classification. Ann Transl Med.; 3(7): 95.

11. Heldin $\mathrm{CH}$, and Westermark B. (1990) : Platelet-derived growth factor: Mechanism 0 action and possible in vivo function. Cell Regul; 1:555

12. Reigstad LJ, Varhaug JE, Lillehaug JR. (2005) : Structural and functional specificities of PDGF-C and PDGF-D, the novel members of the plateletderived growth factors family. FEBS J; 272:572.

13. Blume-Jensen $P$, and Hunter T. (2001) : Oncogenic kinase signalling. Nature. 20; 411:355-365.

14. Heinrich MC, Corless CL, Duensing $A$, McGreevey $L$, et al (2003) : PDGFRA activating mutations in gastrointestinal stromal tu- 
mors. Science., 299: 708-

710.

15. Toffalini F, and Demoulin JB (2010) : New insights into the mechanisms of hematopoietic cell transformation by activated receptor tyrosine kinases. Blood., 116: 2429-2437.

16. Lokker NA, Sullivan CM, Hollenbach SJ, Israel MA, et al. (2002) : Platelet-derived growth factor (PDGF) autocrine signaling regulates survival and mitogenic pathways in glioblastoma cells: evidence that the novel PDGF-C and PDGF$D$ ligands may play a role in the development of brain tumorCancer Res.; 62 (13):3729 -35

17. Smith J, Yang W, Oian J, Hosek S, et al., A(2000) : mplification of the PlateletDerived Growth Factor Receptor-A (PDGFRA) Gene Occurs in Oligodendrogliomas with Grade IV Anaplastic Features. Journal of Neuropathology and Exper- imental Neurology.; 59(6 ): 495-50.

18. Westermark B. (2014) : Platelet-derived growth factor in glioblastoma-driver or biomarker? Ups J Med Sci. Nov; 119(4): 298-305.

19. Pietras K, Sjöblom $T$, Rubin $\mathrm{K}$, Heldin $\mathrm{CH}$, et al . (2003) : PDGF receptors as cancer drug targets Cancer Cell. May;3(5):4394.

20. Wei Liu $k$, and Cheng SH. (2011) : Platelet-derived growth factor receptor alpha in glioma: a bad seed. Chin J Cancer. Sep; 30(9): 590-602.

21. Allan K, Richard C, Jordan K, Ang $L$ et al. (2000) : Overexpression of Cyclin $A$ and Cyclin B1Proteins in Astrocytomas Katherine Allan;. Arch Pathol Lab Med.; Vol 124.

22. Hanahan, D. and Weinberg, R. A. (2011) : Hallmarks of cancer: the next generaMANSOURA MEDICAL JOURNAL 
tion. Cell. 144, 646-674.

23. Musgrove E, C. (2011) : Caldon E, Barraclough J, Stone $A$ \& Sutherland $R$. Cyclin $D$ as a therapeutic target in cancer. Nature Reviews Cancer. 2011; 11:558-572.

24. Santarius, T., Shipley, J., Brewer, D., Stratton, M. R., et al. (2010) : A census of amplified and overexpressed human cancer genes. Nature Rev. Cancer 10, 59-64.

25. Arnold, A, and Papanikolaou, A. (2005) : Cyclin D1 in breast cancer pathogenesis. J. Clin. Oncol. 23, 4215-4224.

26. Sallinen $S$, Sallinen $P$, Kononen J, Syrjäkoski K, et al. (1999) : Cyclin D1 expression in astrocytomas is associated with cell proliferation activity and patient prognosis, the journal of pathology.; 188 289-293

Vol. 44, No. 1 \& 2 Jan. \& April, 2015
27. Bosone I, Cavalla P, ChiadòPiat L, Vito ND, et al. (2001) : Cyclin D1 expression in normal oligodendroglia and microglia cells: its use in the differential diagnosis of oligodendrogliomas. Neuropathology.; 21 (3):155-61.

28. Levine AJ, Momand J, Finlay CA. (1991) : The p53 tumor suppressor gene. Nature; 351:453-6).

29. Ohgaki $H$, Dessen $P$, Jourde $B$, Horstmann $S$, , et al. (2004) : Genetic pathways to glioblastoma: a population-based study. Cancer Res.; 64:6892-6899.

30. Ren ZP, Olofsson T, Qu M, Hesselager G, et al . Molecular genetic analysis of p53 intratumoral heterogeneity in human astrocytic brain tumors. J Neuropathol Exp Neurol. 66(10):94454.

31. Martinho $O$, Longatto-Filho A, Lambros M, C Pinheiro A, et al. (2009) : Fenwick 
$\mathrm{K}$,Ashworth A, Reis-Filho, Lopes $\mathrm{J}$ and Reis R. Expression, mutation and copy number analysis of platelet-derived growth factor receptor A (PDGFRA) and its ligand PDGFA in gliomas. $\mathrm{Br} \mathrm{J}$ Cancer. 15; 101(6): 973-982.

32. Arshad H, Ahmad Z, Hasan SH. (2010) : Gliomas: correlation of histologic grade, Ki67 and p53 expression with patient survival. Asian Pac J Cancer Prev.; 11 (6):1637-40.

33. Zagzag D, Blanco C, Friedlander DR, Miller DC, et al: (2003) : Expression of p27KIP1 in human gliomas: relationship between tumor grade, proliferation index, and patient survival. Hum Pathol, 34: 48-53.

34. Weller, M (2012) : Management of gliomas: relevance of molecular markers for clinical practice. European Association of NeuroOncology Magazine, 2(1):6-10.
35. Cheng $\mathrm{Y}, \mathrm{Ng} \mathrm{HK}$, Zhang SF, et al. (1999) : Genetic alterations in pediatric high grade astrocytomas. Human Pathol; 30:1284-90.

36. Shih, A, Dai C, Hu X, Rosenblum $M$, et al. (2004) : Dose-Dependent Effects of Platelet-Derived Growth Factor-B on Glial Tumorigenesis. Cell and Tumor Biology.

37. Raica $M$ and Cimpean $A$. (2010) : Platelet-Derived Growth Factor (PDGF)/ PDGF Receptors (PDGFR) Axis as Target for Antitumor and Antiangiogenic Therapy Pharmaceuticals.; 3:572-599.

38. Heldin C-H (2013) : Targeting the PDGF signaling pathway in the treatment of non-malignant diseases. $\mathrm{J}$ Neuroimmune Pharmacol.

39. Dunn I, Heese O, Black P. (2000) : Growth factors in glioma angiogenesis: FGFs, PDGF, EGF, and TGFs Journal of NeuroMANSOURA MEDICAL JOURNAL 
Oncology 200 : 121-137.

40. Ribom D, J Andræ, M Frielingsdorf, M Hartman, et al. (2002) : Prognostic value of platelet derived growth factor? receptor expression in grade 2 astrocytomas and oligoastrocytomas J Neurol Neurosurg Psychiatry .; 72:782-787

41. LaRochelle WJ, Jeffers $M$, McDonald WF, et al. (2001) : PDGF-D, a new protease-activated growth factor. Nat Cell Biol.; 3:517-21

42. Shen J, Vil MD, Prewett M, Damoci C, et al. (2009) : Development of a fully human anti-PDGFR? antibody that suppresses growth of human tumor xenografts and enhances antitumor activity of an anti-VEGFR2 antibody. Neoplasia.; 11: 594-604.

43. Bao $\mathrm{S}, \mathrm{Wu} Q$, Sathornsumetee $S$, et al. (2006) : Stem cell-like glioma cells pro- mote tumor angiogenesis through vascular endothelial growth factor. Cancer Res. 66(16) :7843-7848.

44. Guo P, Hu B, Gu W, Xu L, et al. (2003) : PlateletDerived Growth Factor-B Enhances Glioma Angiogenesis by Stimulating Vascular Endothelial Growth Factor Expression in Tumor Endothelia andby Promoting Pericyte Recruitment American Journal of Pathology.;162: 4,

45. Hede SM, Hansson I, Afink GB, Eriksson A, et al. (2009) : GFAP promoter driven transgenic expression of PDGFB in the mouse brain leads to glioblastoma in a Trp53 null background. Glia.; 57:1143-53

46. Gorovets D, Kannan K, Shen R, et al. (2012) : IDH mutation and neuroglial developmental features define clinically distinct subclasses of lower grade diffuse astrocytic glioma. Clin Can- 
cer Res. 2012;18:249-250

\section{Ehrmann JJ, Kolyr Z, Vojte-}

sek B. (1997) : Prognostic factors in astrocytomas: relationship of $\mathrm{p} 53, \mathrm{MDM} 2$, BCL-2 and PCNA immunohistochemical expression to tumor grade and overall patient survival. Neoplasma.; 44:299-304

48. Rainov NG, Dobberstein KU, Bahn H, et al. (1997) : Prognostic factors in malignant glioma: influence of the overexpression of oncogene and tumorsuppressor gene products on survival. J Neurooncol. $1997 ; 35: 13-28$.

49. Ranuncolo SM, Varela M, Morandi A, Lastiri J, et al. (2004) : Prognostic value of Mdm2, p53 and p16 in patients with astrocytomas. J Neurooncol. ; 68(2):11321.

50. Nieder C., Petersen S., Petersen C., Thames H. D. (2000) : The challenge of p53 as prognostic and pre- dictive factor in gliomas. Cancer Treat. Rev., 26: 6773, .

51. Nayak A, Ralte A, Sharma $M$, Singh V, et al. (2004) : p53 protein alterations in adult astrocytic tumors and oligodendrogliomas. Neurology India.; 52 ( 2)

52. Bartkova J, Lukas J, Muller H, Lutzhoft D, et al. (1997) : Cyclin D1protein expression and function in human breast cancer. Int J Cancer. 1994;57: 353-361.

53. Jiang W, Kahn SM, Tomita N, Zhang Y, et al. (1992) : Am(plification and overexpression of the human cyclin D gene in esophageal cancer. Cancer Res.; 52:2980-2983.

54. Bartkova J, Lukas J, Muller H, Strauss M, et al. (1995) : Abnormal patterns of D-type cyclin expression and $\mathrm{G} 1$ regulation in human head and neck cancer. Cancer Res.; 55:949-956.

MANSOURA MEDICAL JOURNAL 
40 PDGFRA EXPRESSION IN ADULT ASTROCYTOMA etc...

55. Musgrove E, C. Caldon E, Barraclough J, Stone A et al. Cyclin $D$ as a therapeutic target in cancer Nature Reviews Cancer 11, 558572.

56. Stupp $R$, Mason WP, van den Bent MJ, Weller M, et al. (2005) : Radiotherapy plus concomitant and adjuvant temozolomide for glioblastoma. $\mathrm{N}$ Engl $\mathrm{J}$ Med.; 352:987-96.

57. Cavalla P, Dutto A, Piva R, Richardi P, et al. (1998) : Cyclin Diexpression in gliomas. Acta Neuropathol.; 95:131-135.

58. Tan PG1, Xing Z, Li ZQ. (2004) : Expression of cyclin D1 in brain gliomas and its significance. $\mathrm{Ai}$ Zheng.; 23(1):63-5
59. Louis DN. (1997) : A molecular genetic model of astrocytoma histopathology. Brain Pathol. 7; 755-764.

60. Hesselager G, Uhrbom L, (2003) : Westermark B, and Nistér M Complementary Effects of Plateletderived Growth Factor Autocrine Stimulation and p53 or Ink4a-Arf Deletion in a Mouse Glioma Mode. Cancer research.; 63, 15

61. Mishina $T$, Dosaka-Akita $H$, Kinoshita I, Hommura F, et al. (1999) : Cyclin D1 expression in non-smallcell lung cancers: its association with altered p53 expression, cell proliferation and clinical outcome. $\mathrm{Br} \mathrm{J}$ Cancer. 1999; 80(8): 1289-1295. 